\title{
Inhomogeneities in Superconductors Described by the Two-Component Model
}

\author{
J. KRZYSZCZAK*, T. Domański And K.I. WYSOKIŃSKi \\ Institute of Physics and Nanotechnology Centre, M. Curie-Skłodowska University \\ Pl. M. Curie-Skłodowskiej 1, 20-031 Lublin, Poland
}

\begin{abstract}
We study fluctuations of the superconducting order parameter of $s$-wave symmetry caused by nonmagnetic disorder present both in the boson and fermion subsystems using the Bogoliubov-de Gennes equations. In particular, we are interested if some features of the $d$-wave model persist for $s$-wave symmetry as well. These are: positive correlations between positions of impurities and the magnitude of energy gap, the homogeneity of the local density of states at low energies, nanoscale inhomogeneity of the coherence peak positions and their non-uniform height. We have found that spatial variations of $s$-wave order parameter are positively correlated with the positions of impurities. Such behavior has been observed in scanning tunneling measurements on $d$-wave symmetry BSCCO-2122 superconductors.
\end{abstract}

PACS numbers: 71.10.Fd, 74.20.-z, 74.81.-g

\section{Introduction}

Analysis of how disorder affects the superconductivity has been a matter of great concern for about last 50 years, both in experimental and theoretical studies [1]. Consensus about why high temperature superconductors (HTS) are so insensitive to non-magnetic disorder, despite the fact that theory suggests its pair breaking character for a $d$-wave symmetry case $[2,3]$, has not yet emerged. This problem has been given new twist recently with a series of experiments on the cuprate superconductors $[4,5]$. In particular, measurements of the scanning tunneling spectroscopy (STS) for $\mathrm{Bi}_{2} \mathrm{Sr}_{2} \mathrm{CaCu}_{2} \mathrm{O}_{8+x}$ revealed existence of the large intrinsic spatial inhomogeneities. Similar, even though not so precise, data have been reported on pnictides [6-8].

In HTS the differences in positions of the coherence peak(s) in the differential conductance (being a measure of the superconducting gap) have been observed to vary by a factor of $2 \sim 3$ over a few lattice constants. Detailed analysis of the experimental data indicated also other interesting issues such as: (i) homogeneity at low [5] and very high energies, (ii) asymmetry of the differential conductance between positive and negative bias, (iii) and a surprising positive correlation of the dopant atoms' position with the spectral gap in the local density of states (LDOS) [9].

Our purpose here is to carry out theoretical calculations on highly disordered, short coherence length, $s$-wave superconductor described by the two component boson-fermion model [10] using the mean field approximation and Bogoliubov-de Gennes equations. Similar numerical studies for the one component $t-J$ models have been investigated by a number of groups considering the

* corresponding author; e-mail: jkrzyszczak@kft.umcs.lublin.pl $s$-wave [11] and $d$-wave symmetries [12-16]. In the two-component scenario we shall extend their and our previous analysis [17] exploring the several possible kinds of inhomogeneities affecting the system. The charge-exchange driven superconductivity of the boson-fermion model (1) and its relevance to the high temperature cuprates as well as other exotic quantum superfluids has been so far discussed by the number of groups studying homogeneous [18] or impure systems [19, 20].

\section{The model and approach}

The boson-fermion model which describes the mixture of tightly bound local pairs (hard-core bosons) coexisting with the itinerant holes (fermions) can be represented by the following Hamiltonian [10]:

$$
\begin{gathered}
\hat{H}^{\mathrm{BF}}=\sum_{i \sigma}\left(V_{i}^{\mathrm{imp}}-\mu\right) \hat{c}_{i \sigma}^{\dagger} \hat{c}_{i \sigma}+\sum_{i}\left(E_{i}^{\mathrm{B}}-2 \mu\right) \hat{b}_{i}^{\dagger} \hat{b}_{i} \\
+\sum_{i, j, \sigma} t_{i j} \hat{c}_{i \sigma}^{\dagger} \hat{c}_{j \sigma}+\sum_{i} g_{i}\left(\hat{b}_{i}^{\dagger} \hat{c}_{i \downarrow} \hat{c}_{i \uparrow}+\hat{b}_{i} \hat{c}_{i \uparrow}^{\dagger} \hat{c}_{i \downarrow}^{\dagger}\right)
\end{gathered}
$$

where $i$ and $j$ denote the sites of the two dimensional square lattice. $\hat{c}_{i, \sigma}$ and $\hat{c}_{i, \sigma}^{\dagger}\left(\hat{b}_{i}\right.$ and $\left.\hat{b}_{i}^{\dagger}\right)$ are fermionic (bosonic) annihilation and creation operators, $\mu$ stands for the common chemical potential which ensures the charge conservation. We denote the hopping integral of itinerant fermions by $t_{i j}$ and assume site-dependent energies $E_{i}^{\mathrm{B}}$ of the local pairs and their coupling $g_{i}$ to fermions. We take into account the hopping integrals to the nearest neighbor $(t)$ and next nearest neighbor sites $\left(t^{\prime}\right)$ only. Applying the standard Hartree-Fock-Bogoliubov decoupling $\hat{b}_{i}^{\dagger} \hat{c}_{i, \downarrow} \hat{c}_{i, \uparrow} \simeq$ $\left\langle\hat{b}_{i}^{\dagger}\right\rangle \hat{c}_{i, \downarrow} \hat{c}_{i, \uparrow}+\hat{b}_{i}^{\dagger}\left\langle\hat{c}_{i, \downarrow} \hat{c}_{i, \uparrow}\right\rangle$ decomposes the Hamiltonian (1) into separate boson and fermion parts [21] $\hat{H}^{\mathrm{BF}}=$ $\hat{H}^{\mathrm{B}}+\hat{H}^{\mathrm{F}}$, where $\hat{H}^{\mathrm{B}}=\sum_{i}\left[\left(E_{i}^{\mathrm{B}}-2 \mu\right) \hat{b}_{i}^{\dagger} \hat{b}_{i}+\hat{b}_{i}^{\dagger} \chi_{i}+\right.$ $\left.\hat{b}_{i} \chi_{i}^{*}\right]$ with bosonic order parameter $\chi_{i}=g_{i}\left\langle\hat{c}_{i \downarrow} \hat{c}_{i \uparrow}\right\rangle$ 
and $\hat{H}^{\mathrm{F}}=\sum_{i, j, \sigma} t_{i j} \hat{c}_{i \sigma}^{\dagger} \hat{c}_{j \sigma}+\sum_{i \sigma}\left(V_{i}^{\mathrm{imp}}-\mu\right) \hat{c}_{i \sigma}^{\dagger} \hat{c}_{i \sigma}+$ $\sum_{i}\left(\Delta_{i}^{*} \hat{c}_{i \downarrow} \hat{c}_{i \uparrow}+\Delta_{i} \hat{c}_{i \uparrow}^{\dagger} \hat{c}_{i \downarrow}^{\dagger}\right)$. For a given realization of disorder we compute at every site the local number of bosons $\left\langle\hat{b}_{i}^{\dagger} \hat{b}_{i}\right\rangle=1 / 2-\left[\left(E_{i}^{\mathrm{B}}-2 \mu\right) / 4 \epsilon_{i}\right] \tanh \left(\epsilon_{i} / k_{\mathrm{B}} T\right)$ and $\left\langle\hat{b}_{i}\right\rangle=-\left(\chi_{i} / 2 \epsilon_{i}\right) \tanh \left(\epsilon_{i} / k_{\mathrm{B}} T\right)$ with $\Delta_{i}=g_{i}\left\langle\hat{b}_{i}\right\rangle$ and $\epsilon_{i}=\sqrt{\left(E_{i}^{\mathrm{B}} / 2-\mu\right)^{2}+\left|\chi_{i}\right|^{2}}$.

The fermion part has a BCS-type structure and can be exactly diagonalized [22] by the Bogoliubov-Valatin transformation. For the inhomogeneous systems one has to solve self-consistently the following Bogoliubovde Gennes equations [22, 23]

$$
\begin{aligned}
& \sum_{j}\left(\begin{array}{c}
t_{i j}+\left(V_{i}^{\mathrm{imp}}-\mu\right) \delta_{i j} ; \Delta_{i} \delta_{i j} \\
\Delta_{i}^{*} \delta_{i j} ;-t_{i j}-\left(V_{i}^{\mathrm{imp}}-\mu\right) \delta_{i j}
\end{array}\right)\left(\begin{array}{c}
u_{j}^{l} \\
v_{j}^{l}
\end{array}\right) \\
& =E^{l}\left(\begin{array}{c}
u_{i}^{l} \\
v_{i}^{l}
\end{array}\right)
\end{aligned}
$$

determining the energies $E^{l}$ and coefficients $u_{i}^{l}, v_{i}^{l}$. The local density of states $\rho(i, \omega)$ at site $i$ as function of energy $\omega$, local fermion, boson and total carrier densities are in turn expressed by these eigensolutions. We will also study the correlation functions in real space which for arbitrary quantities $f, h$ is defined as [22] $C_{f, h}(R)=$ $\sum_{i} \sum_{j}\left(f_{i}-\bar{f}\right)\left(h_{j}-\bar{h}\right) / \sqrt{\sum_{i}\left(f_{i}-\bar{f}\right)^{2}} \sqrt{\sum_{j}\left(h_{j}-\bar{h}\right)^{2}}$ with $R=|i-j|$.

\section{The results}

Disorder in the two-component model can be taken into account in various ways. Firstly, one can imagine that impurities change local atomic levels, which in our model are represented by $V_{i}^{\mathrm{imp}}$. Secondly, the values of the hopping integrals $t_{i j}$ can fluctuate from site to site, as dislocations or imperfections may appear with doping. Thirdly, the value of boson-fermion coupling $g_{i}$ can be modified. Last, but not least, positions of boson energy levels $E_{i}^{\mathrm{B}}$ could locally change. Suggestions that in vicinity of dopants, lattice distortions could locally modulate the interactions [24] appeared long ago and have recently been explored to predict an enhancement of the critical temperature $T_{\mathrm{c}}[25]$ as a result of the proximity effect.

We focus on the following set of parameters: $g_{0}=1.0 t$, $V_{i}^{\mathrm{imp}}=0.0 t, E_{i}^{\mathrm{B}}=2 \mu+0.58 t$ for the superconducting and $E_{i}^{\mathrm{B}}=2 \mu+10.65 t$ for non-superconducting host. $t$ is our energy unit. The size of the system is $57 \times 61$ sites. We study the effect of impurities on the local value of the order parameter $\Delta_{i}$ and the evolution of the spectral gap in the local density of states in inhomogeneous superconductor. It turns out that there exist a number of correlations, e.g. (i) the values of the gap and positions of impurities $R_{i}$, (ii) the order parameter $\left\langle\hat{b}_{i}\right\rangle$ and positions of impurities, (iii) the values of the gap and height of coherence peaks in local density of states, etc. The autocorrelation function between values of the gap at different points serves as a measure of the coherence length. All
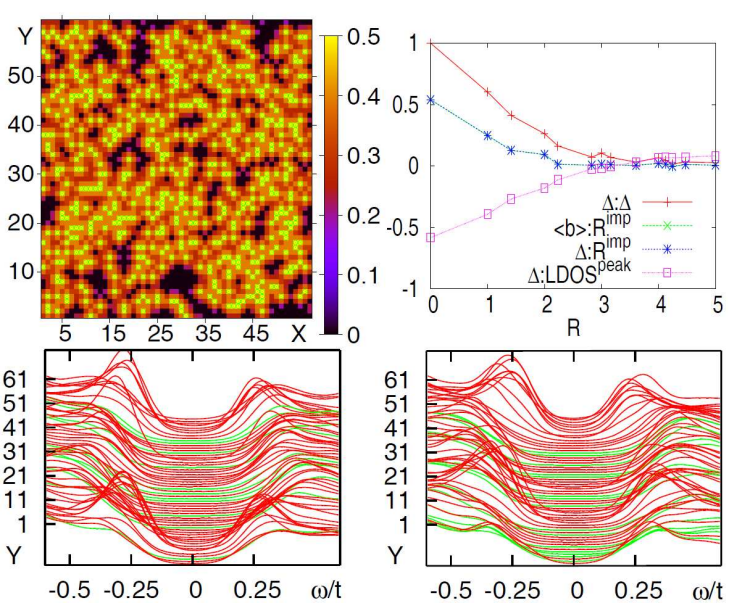

Fig. 1. Map of the order parameter $\Delta_{i}$ (top left panel), the correlation functions $C_{f, h}(R)$ (top right panel) and the STM spectra taken along vertical line at $X=13$ (bottom left panel) and $X=47$ (bottom right panel) for $16 \%$ of "extended" impurities (marked by circles), which influence the positions of $E_{i}^{\mathrm{B}}-2 \mu$ levels of neighboring sites changing them from homogeneous value $10.65 t$ to $0 t$ at the impurity, $0.29 t$ at its nearest neighbor site, $0.425 t$ and $0.5 t$ at still further sites. The next nearest hopping $t^{\prime}=-0.3 t$ and the total concentration of carriers $n_{\text {tot }}=1.4$.
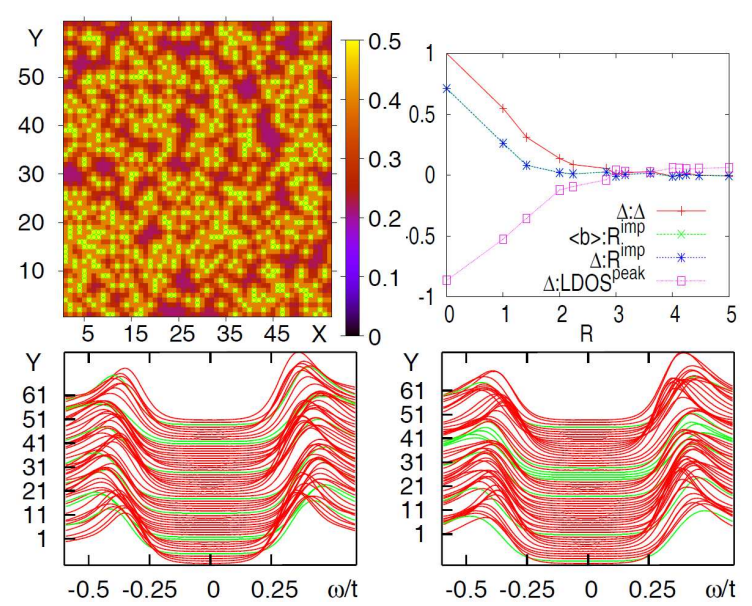

Fig. 2. Map of the order parameter $\Delta_{i}$ (top left panel), the correlation functions $C_{f, h}(R)$ (top right panel) and the STM spectra taken along vertical line at $X=24$ (bottom left panel) and $X=40$ (bottom right panel) for $16 \%$ of "extended" impurities (marked by circles) which influence the positions of $E_{i}^{\mathrm{B}}$ levels of neighboring sites and the local atomic levels $V_{i}^{\mathrm{imp}}$, changing them from homogeneous value $\left(E_{i}^{\mathrm{B}}-2 \mu, V_{i}^{\mathrm{imp}}\right)=(0.58,0.0) t$ to $(0.0,-0.29) t$ at the impurity, $(0.29,-0.145) t$ at its nearest neighbor site, $(0.425,-0.0775) t$ and $(0.5,-0.04) t$ at still further sites. $t^{\prime}=0 t$ and the total concentration of carriers $n_{\text {tot }}=1.3$. 
results presented in Figs. 1 and 2 were obtained for a ground state $(T=0 \mathrm{~K})$.

Figure 1 presents the results for "extended" out-of-plane impurities which modify position of boson level energy $E_{i}^{\mathrm{B}}$ at a central and few neighboring sites of the homogeneous non-superconducting system in a way that supports superconductivity - that is by pushing $E_{i}^{\mathrm{B}}$ closer to the Fermi energy $\mu$. Figure 2 shows the effect of the "extended" impurities, which also introduce the potential scattering $V_{i}^{\text {imp }}$ in the superconducting fermion subsystem. We assume that if impurity modifies the $E_{i}^{\mathrm{B}}$ value by a $\delta$ for an electron pair, then modification for an electron equals to $\delta / 2[19]$.

It is interesting to note that many of features of the two-component model are not sensitive to the symmetry of the order parameter. In Figs. 1 and 2, which are obtained for $s$-wave superconductor the characteristics are qualitatively and even quantitatively the same as for $d$-wave one [17]. These are shapes of correlation function $C_{f, h}$ for the position of the impurity and the value of the gap and the anti-correlation between values of the gap and height of coherence peaks. They both are close to those seen in experiment $(0.3 \sim 0.4)$ [9]. The homogeneity of the local density of states at low bias is again a feature of the boson-fermion model observed for $s$ - and $d$-wave symmetry and also visible in experimental data $[4,5,9]$. Of course the shape of the LDOS is different as it depends on symmetry.

The main assumption of the model necessary to explain many puzzling experimental data is connected with the mechanism leading to the required changes of the boson-fermion model. The calculations similar to those of Altman and Auerbach [26] with oxygen defects taken into account would clarify the issue and make the approach selfcontained.

\section{Summary and conclusions}

We have studied several possible sources of disorder within the boson-fermion model and shown that the observed STS data could be explained within a reasonable assumption that impurities change the position of boson energy levels $E_{i}^{\mathrm{B}}$ moving them towards the Fermi level. In the more realistic case we took into account fluctuations of the atomic levels $V^{\mathrm{imp}}$. These types of disorder provide natural explanation to a number of observations on HTS. In particular one explains: the observed nanoscale inhomogeneity of the superconducting gaps, homogeneity of LDOS at low and very high energies, positive correlation between position of dopant atoms and spectral gap and anti-correlation between values of the gap and height of coherence peaks. The two-component model is of importance to study two channel superfluids in optical lattices [27], where both $s$ - and $d$-wave symmetry spin singlet condensates can easily be obtained. Thus they give the opportunity to check the present ideas and results to much higher accuracy than can be obtained in HTS.

\section{Acknowledgments}

This work has been partially supported by the grant no. N N202 187833.

\section{References}

[1] W. Buckel, R. Hilsch, Z. Phys. 132, 420 (1952).

[2] A.I. Larkin, JETP Lett. 2, 130 (1965).

[3] A.M. Martin, G. Litak, B.L. Györffy, J.F. Annett, K.I. Wysokiński, Phys. Rev. B 60, 7523 (1999).

[4] T. Cren, D. Roditchev, W. Sacks, J. Klein, J.-B. Moussy, C. Deville-Cavellin, M. Laguës, Phys. Rev. Lett. 84, 147 (2000); S.-H. Pan, J.P. O'Neal, R.L. Badzey, C. Chamon, H. Ding, J.R. Engelbrecht, Z. Wang, H. Eisaki, S. Uchida, A.K. Gupta, K.-W. Ng, E.W. Hudson, K.M. Lang, J.C. Davis, Nature (London) 413, 282 (2001); C. Howald, P. Fournier, A. Kapitulnik, Phys. Rev. B 64, 100504(R) (2001); J.E. Hoffman, E.W. Hudson, K.M. Lang, V. Madhavan, H. Eisaki, S. Uchida, J.C. Davis, Science 295, 466 (2002).

[5] K.M. Lang, V. Madhavan, J.E. Hoffman, E.W. Hudson, H. Eisaki, S. Uchida, J.C. Davis, Nature (London) 415, 412 (2002).

[6] O. Millo, I. Asulin, O. Yuli, I. Felner, Z.-A. Ren, X.L. Shen, G.-C. Che, Z.-X. Zhao, Phys. Rev. B 78, 092505 (2008).

[7] Y. Yin, M. Zech, T.L. Williams, X.F. Wang, G. Wu, X.H. Chen, J.E. Hoffman, Phys. Rev. Lett. 102, 097002 (2009).

[8] F. Massee, Y. Huang, R. Huisman, S. de Jong, J.B. Goedkoop, M.S. Golden, Phys. Rev. B 79, 220517(R) (2009).

[9] K. McElroy, Jinho Lee, J.A. Slezak, D.-H. Lee, H. Eisaki, S. Uchida, J.C. Davis, Science 309, 1048 (2005).

[10] J. Ranninger, S. Robaszkiewicz, Physica B 135, 468 (1985).

[11] A. Ghosal, M. Randeria, N. Trivedi, Phys. Rev. Lett. 81, 3940 (1998).

[12] M. Franz, C. Kallin, A.J. Berlinsky, Phys. Rev. B 54 R6897 (1996).

[13] W.A. Atkinson, P.J. Hirschfeld, A.H. MacDonald, Phys. Rev. Lett. 85, 3922 (2000).

[14] A. Ghosal, M. Randeria, N. Trivedi, Phys. Rev. B 63, 020505(R) (2000).

[15] B.M. Andersen, A. Melikyan, T.S. Nunner, P.J. Hirschfeld, Phys. Rev. B 74,060501(R) (2006).

[16] M.M. Maśka, Ż. Śledx́, K. Czajka, M. Mierzejewski, Phys. Rev. Lett. 99, 147006 (2007).

[17] J. Krzyszczak, T. Domański, K.I. Wysokiński, R. Micnas, S. Robaszkiewicz, J. Phys., Condens. Matter 22, 255702 (2010).

[18] R. Micnas, J. Ranninger, S. Robaszkiewicz, Rev. Mod. Phys. 62, 113 (1990); R. Friedberg, T.D. Lee, H.C. Ren, Phys. Rev. B 42, 4122 (1990); V.B. Geshkenbein, L.B. Ioffe, A.I. Larkin, Phys. Rev. B 55, 3173 (1997); L.P. Gor'kov, J. Supercond. 13, 765 (2000); T. Domanski, J. Ranninger, Phys. Rev. B 63, 134505 (2001); A.H. Castro Neto, Phys. Rev. B 64, 104509 (2001). 
[19] Ż. Śledź, M. Mierzejewski, Acta Phys. Pol. A 114, 219 (2008).

[20] G. Pawłowski, S. Robaszkiewicz, Physica A 299, 475 (2001); G. Pawłowski, S. Robaszkiewicz, R. Micnas, Acta Phys. Pol. A 106, 745 (2004).

[21] T. Domański, K.I. Wysokiński, Phys. Rev. B 66, 064517 (2002); T. Domański, J. Ranninger, K.I. Wysokiński, Acta Phys. Pol. B 34, 493 (2003).

[22] T.S. Nunner, B.M. Andersen, A. Melikyan, P.J. Hirschfeld, Phys. Rev. Lett. 95, 177003 (2005).

[23] J. Krzyszczak, T. Domański, K.I. Wysokiński, Acta Phys. Pol. A 114, 165 (2008).
[24] I.N. Khlyustikov, A.I. Buzdin, Adv. Phys. 36, 271 (1987); A.I. Larkin, Sov. Phys. JETP 31, 784 (1970).

[25] I. Martin, D. Podolsky, S.A. Kivelson, Phys. Rev. B 72, 060502(R) (2005).

[26] E. Altman, A. Auerbach, Phys. Rev. B 65, 104508 (2002).

[27] I. Bloch, J. Dalibard, W. Zwerger, Rev. Mod. Phys. 80, 885 (2008). 